\title{
Passiv in deutschsprachigen Chats. Eine Korpusanalyse.
}

\author{
Petra M. Vogel (Bern)
}

\begin{abstract}
In this paper I will investigate some passive phenomena in German chat communication including personal and impersonal passive, passive-like constructions and the use of the "dummy" pronoun es. Furthermore, I will compare the results of this study with other empirical works on passive in contemporary German (Brinker 1971 and Schoenthal 1976) thereby discussing whether chat language can be categorized as "spoken German".
\end{abstract}

\section{$1 \quad$ Einleitung}

Im Allgemeinen wird in der linguistischen Forschung von einem Gegensatz Schriftsprache oder geschriebene Sprache versus Sprechsprache oder gesprochene Sprache ausgegangen. Dabei entsteht der Eindruck, dass die Art der Informationsübermittlung (schriftlich versus mündlich) automatisch an eine bestimmte Kommunikationssituation (öffentlich versus privat) sowie einen bestimmten Sprachgebrauch (elaboriert versus nicht-elaboriert) geknüpft ist. Demgegenüber weisen Koch/Oesterreicher (1994: 587) darauf hin, dass zwischen zwei Ebenen, nämlich Medium und Konzeption, sowie entsprechend zwischen medialer Schriftlichkeit/Mündlichkeit und konzeptioneller Schriftlichkeit/Mündlichkeit unterschieden werden muss.

Während mediale Schriftlichkeit/Mündlichkeit eine Dichotomie darstellt, handelt es sich im Falle von konzeptioneller Schriftlichkeit/Mündlichkeit um ein Kontinuum, das sowohl durch die Kommunikationsbedingungen als auch durch die Versprachlichungsstrategien bestimmt ist. Hinsichtlich der Kommunikationssituation werden den beiden Polen des Kontinuums die Begriffe "Nähe" (konzeptionelle Mündlichkeit) und "Distanz" (konzeptionelle Schriftlichkeit) zugeordnet, die durch Parameter wie Öffentlichkeit, Monolog/Dialog, Vertrautheit der Kommunikationspartner, Emotionalität, Situations- und Handlungseinbindung, Spontaneität, Themenfixierung usw. bestimmt sind (Koch/Oesterreicher 1994: 588). In Bezug auf Versprachlichungsstrategien zeichnet sich der Mündlichkeitspol etwa durch geringere Informationsdichte, Kompaktheit, Komplexität, Elaboriertheit usw. aus (Koch/Oesterreicher 1985: 23, nach Dürscheid 2002: 53). Deshalb finden sich hier gehäuft z.B. "fehlerhafte[r] Satzbau, Flexionsbrüche, Dialektismen, umgangssprachliche Ausdrücke, Ellipsen, Selbstkorrekturen, Gesprächspartikeln" (Dürscheid 2002: 29).

Prinzipiell ist im Chat mediale Schriftlichkeit mit konzeptioneller Mündlichkeit verknüpft (s.a. Dürscheid 2002: 54), was sich auch darin niederschlägt, dass der Chat als "getipptes Gespräch" (Storrer 2001: 462) oder als "geschriebene Umgangssprache" (Kilian 2001) 
bezeichnet wird. Einschränkend ist jedoch zu bemerken, dass konzeptionelle Mündlichkeit zwar in Hinblick auf die sprachlichen Strukturen gelten mag, auf der Ebene der Kommunikationssituation scheint aber keine eindeutige Zuordnung zu "Nähe" oder "Distanz" möglich. So sprechen zwar z.B. die Merkmale Spontaneität oder Dialog für eine Zuordnung zum Nähepol, maximale Öffentlichkeit oder geringe Vertrautheit der Teilnehmer miteinander jedoch dagegen (Dürscheid 2002: 51f.). M.E. soll aber durch die konzeptionell mündliche Sprache kommunikationssituative "Nähe" simuliert werden, so dass der Chat letztendlich doch sowohl in Hinblick auf Kommunikationsbedingungen als auch auf Versprachlichungsstrategien als konzeptionell mündlich eingeordnet werden kann. Wenn hier im Folgenden zwischen privater und öffentlicher Kommunikationssituation unterschieden wird, gilt der Chat als privat.

Diese Zuordnung zum Bereich "konzeptionelle Mündlichkeit" soll hier anhand des Phänomens Passiv genauer untersucht werden. Dabei liegt mit den empirischen Arbeiten von Brinker 1971 und Schoenthal 1976 zum Passiv in der deutschen Gegenwartssprache eine gute Vergleichsbasis vor. Brinker 1971 stützt seine Untersuchungen zum Passiv im Gegenwartsdeutschen auf (medial geschriebene) Texte aus den Bereichen Dichtung, Trivialliteratur, wissenschaftliche und populärwissenschaftliche Literatur, Zeitungen und Gebrauchsliteratur (a.a.O. 19f.). Alle Texte können also dem Bereich der konzeptionellen Schriftlichkeit zugeordnet werden. Schoenthal 1976 beschäftigt sich zwar mit (medial) gesprochener Sprache, ihr Material aus den Bereichen Reportage, Bericht, Vortrag, Interview, Informationsgespräch und Diskussion (a.a.O. 151-165) ist jedoch zwischen den Polen konzeptionelle Schriftlichkeit und Mündlichkeit angesiedelt, denn "die Sprecher der Texte sind dafür überwiegend routiniert, bzw. speziell vorbereitet" (a.a.O. 150). Die Untersuchungen zu Passiv im Chat ergänzen somit die vorliegende Datenlage um den Bereich der konzeptionellen Mündlichkeit. Zu erwarten ist, dass die Ergebnisse näher bei Schoenthal 1976 als bei Brinker 1971 liegen.

Im Folgenden wird zuerst die Datenbasis vorgestellt (Abschnitt 2) sowie eine Charakterisierung der $\mathrm{zu}$ untersuchenden Konstruktionen gegeben (Abschnitt 3). Anschließend wird das Korpus im Rahmen von persönlichem (Abschnitt 4) und unpersönlichem Passiv (Abschnitt 5) betrachtet, d.h. Passiv mit bzw. ohne Subjekt. Dem folgt ein Exkurs zum "Platzhalter"-es (Abschnitt 6) sowie ein Kapitel zu Passivvarianten (Dativpassiv usw.) (Abschnitt 7). Die Arbeit schließt mit einer Zusammenfassung der wichtigsten Ergebnisse (Abschnitt 8).

\section{Datenbasis}

Als Datenbasis wurden sechs Internet Relay Chat (IRC)-Logfiles verwendet, die vom 5. bis 19.02.2001 am Germanistischen Institut der Universität Göteborg aufgezeichnet wurden (s. den Artikel von Christiane Pankow in diesem Band). Die Logfiles stammen aus den Channels "Berlin", "Hamburg", "Köln", "Linux.de", "München" und "Münster", also inhaltlich gesehen aus fünf regional und einem technisch orientierten Channel. Für die Untersuchung wurden 
offensichtliche Tipp- und Orthographiebesonder-heiten beseitigt sowie Satzzeichen eingefügt, da diesbezügliche Abweichungen für die vorliegende Untersuchung nicht von Relevanz sind.

Die Gesamtwortzahl beträgt rund 173.000, die Anzahl der Gesamtfinita liegt bei etwa 20.000 (stichprobenartige Ermittlung). Insofern entspricht mein Korpus größenmäßig etwa dem von Schoenthal 1976 (s. u.).

\begin{tabular}{|c|c|c|c|}
\hline & $\begin{array}{c}\text { Brinker 1971 } \\
\text { Konzeptionell } \\
\text { schriftliche Sprache }\end{array}$ & $\begin{array}{c}\text { Schoenthal 1976 } \\
\text { Konzeptionell } \\
\text { schriftl.-mündl. } \\
\text { Sprache }\end{array}$ & $\begin{array}{c}\text { Chat } \\
\text { Konzeptionell } \\
\text { mündliche } \\
\text { Sprache }\end{array}$ \\
\hline Satzzahl & 15.000 & 8000 & - \\
\hline Gesamtwortzahl & - & 134.000 & 173.000 \\
\hline Finita & 204.000 & 14.000 & 20.000 \\
\hline $\begin{array}{c}\text { Anteil der Finita } \\
\text { an der } \\
\text { Gesamtwortzahl }\end{array}$ & - & $\mathbf{1 0 \%}$ & $\mathbf{1 2 \%}$ \\
\hline Passive & 14.000 & 1200 & 380 \\
\hline $\begin{array}{c}\text { Anteil der Passive } \\
\text { an den Finita }\end{array}$ & $\mathbf{7 \%}$ & $\mathbf{9 \%}$ & $\mathbf{2 \%}$ \\
\hline
\end{tabular}

Hier bestätigen sich außerdem zwei wichtige Tendenzen hinsichtlich sprachlicher Strukturen im Bereich konzeptioneller Mündlichkeit (vgl. Schoenthal 1976: 217f.):

Je näher ein Text am Mündlichkeitspol angesiedelt ist, desto höher ist der Anteil der finiten Verben an der Gesamtwortzahl. Dies ist hier ganz klar gegeben mit einem Anteil von 10\% im Schoenthal-Korpus versus $12 \%$ im Chat-Korpus. Es ist anzunehmen, dass dies mit der durchschnittlich höheren Satzzahl und geringeren Anzahl von Wörtern pro Satz zusammenhängt, je privater eine Kommunikationssituation ist (s.a. Schoenthal 1976: 216).

Umgekehrt sinkt der Anteil der Passive an den Finita mit zunehmender Nähe zum Mündlichkeitspol, was sich deutlich an der Differenz von 9\% (Schoenthal-Korpus) versus 2\% (Chat-Korpus) zeigt. Dies hängt damit zusammen, dass das Passiv als markierte Form komplexer ist als das Aktiv und deshalb im Bereich typisch mündlicher Sprache gemieden wird (Schwitalla 1997: 105 nach Ochs 1979: 69f.). Darauf, dass das Passiv unter öffentlichen bzw. weniger privaten Kommunikationsbedingungen häufiger ist, hat außerdem Pape-Müller (1980: 147) hingewiesen: "[...] der Grund ist, weil in den Texten aus diesen Bereichen das Agens in den Hintergrund tritt, oft gleich bleibt, und es nur auf die reinen Vorgänge ankommt $[\ldots] "$. 


\section{Charakterisierung der zu untersuchenden Passivvorkommen}

Primär handelt es sich um eine Untersuchung des kanonischen Passivs, d.h. um Prädikate der Form sein bzw. werden + Partizip Perfekt. Der Einfachheit halber wird für die Aussortierung auf die bei Brinker (1971: 27f.) genannten Kriterien zurückgegriffen:

a) Der Passivsatz steht mit einem Aktivsatz in Beziehung.

b) Die semantischen Verhältnisse zwischen beiden Sätzen müssen annähernd gleich sein.

c) Die Sätze müssen grammatisch korrekt sein.

d) Beide Sätze müssen ein Prädikat mit demselben Verb aufweisen.

e) Der Aktivsatz muss ein Subjekt enthalten, das nicht gleichzeitig Subjekt des Passivsatzes sein darf.

Teilweise kommt es jedoch zu Konflikten mit formal ähnlichen Konstruktionen, allerdings weniger beim werden-Passiv (das auch als Vorgangspassiv bezeichnet wird) als beim seinoder Zustandspassiv, da hier besonders der Übergang zum Prädikativum, d.h. Kopula + prädikatives Adjektiv, fließend ist. Schoenthal (1976: 27) merkt dementsprechend an, dass Grenzfälle häufig sind. So wird erstaunt in er ist erstaunt von Helbig (1968: 142) als lexikalisiertes Partizip (und damit als Adjektiv und die ganze Konstruktion als Prädikativum), von Brinker (1971: 121) jedoch als partizipialer Teil der Passivperiphrase eingestuft. Eindeutig ausgeschlossen sind nur Fälle, in denen es kein vollständiges Paradigma gibt (wie in ich bin überfragt, da es keinen Infinitiv überfragen gibt), oder wenn die Lexikalisierung zu einer eindeutigen semantischen Wegentwicklung geführt hat (wie in das ist bekannt, das nichts (mehr) mit etw. bekennen zu tun hat) (Schoenthal 1976: 27f.). Im Falle einer solchen Wegentwicklung erfolgt die Perfektbildung außerdem wie bei "normalen" Adjektiven mit geworden anstelle von worden (das ist bekannt geworden) (a.a.O. 28).

Verwechslungsmöglichkeiten bestehen auch mit dem Perfekt oder Plusquamperfekt, wenn es mit dem Hilfsverb sein gebildet ist und damit rein äußerlich dem Präsens bzw. Präteritum des sein-Passivs ähnelt: das Feuer ist aufgelodert (Perfekt) vs. das Feuer ist gelöscht (sein-Passiv im Präsens) (s. Hentschel/Weydt 1994: 122). Nur beim sein-Passiv ist jedoch die Hinzufügung von worden möglich: das Feuer ist gelöscht worden (Passiv) vs. *das Feuer ist aufgelodert worden (Tempus) (a.a.O.). Außerdem ist beim sein-Passiv die Umwandlung in ein werden-Passiv im Präsens möglich, ohne dass ein ungrammatikalischer Satz entsteht: das Feuer wird gelöscht vs. *das Feuer wird aufgelodert (a.a.O.). Umgekehrt kann unter denselben Bedingungen ein Perfekt durch ein Präsens ersetzt werden: die Blume verblüht $(<$ die Blume ist verblüht) vs. * die Flasche öffnet (< die Flasche ist geöffnet) (Schoenthal 1976: 29).

Für das vorliegende Korpus und für die Kategorisierung als sein-Passiv ist entscheidend, dass

a) ein Bezug zu einem Aktivsatz besteht,

b) eine Hinzufügung von worden möglich ist bzw. eine Umformung in ein präsentisches werden-Passiv, und

c) die prinzipielle Möglichkeit einer Agenshinzufügung besteht. 
Ist die Agenshinzufügung realisiert, wird grundsätzlich von einer Passiv-Konstruktion ausgegangen (vgl. auch Schoenthal 1976: 106).

Im Chat-Korpus finden sich also vor allem typische Passive, weniger typische Konstruktionen sind nicht aufgenommen.

Brinker (1971: 76) zählt zum sein-Passiv auch Formen, die nur zu einem Satz mit Reflexivum eine Konverse eröffnen (obwohl hier Kriterium 5. nicht erfüllt ist: "Der Aktivsatz muss ein Subjekt enthalten, das nicht gleichzeitig Subjekt des Passivsatzes sein darf"; s.o.). So kann ein Satz wie die Materie [war] im Anfang im Weltall gleichmäßig verteilt nicht zurückgeführt werden auf *man hat die Materie im Anfang im Weltall gleichmäßig verteilt, sondern nur auf eine Reflexivkonstruktion die Materie hat sich im Anfang im Weltall gleichmäßig verteilt (Brinker 1971: 77). Schoenthal schließt solche Konstruktionen vom kanonischen sein-Passiv aus und betrachtet sie gesondert (z.B. a.a.O. 191f.). In die vorliegende Untersuchungen fließen diese Formen überhaupt nicht ein, weshalb ich mit meinen Kriterien für das seinPassiv näher bei Schoenthal als bei Brinker liege.

Die Passivformen im Korpus werden in Anlehnung an Brinker 1971 in sechs Kategorien aufgeteilt (a.a.O. 29, 71). Dabei ergeben sich 3 Hauptkategorien mit je 2 Unterkategorien, von denen (1) jeweils mit Agenshinzufügung, (2) ohne Agenshinzufügung bedeutet. Die drei Hauptkategorien beziehen sich im Falle von (I) auf ein persönliches Passiv, d.h. mit Subjekt (bei Schoenthal 1976: 24 "E1" für "Ergänzung im Nominativ"), im Falle von (II) und (III) auf das so genannte unpersönliche oder subjektlose Passiv (bei Schoenthal 1976: 181 "Passivkonstruktionen ohne E1"). (II) wird von intransitiven Verben mit Objekt gebildet, (III) von intransitiven Verben ohne Objekt bzw. von absolut gebrauchten transitiven Verben. In der Notation von Brinker (a.a.O. 29, 71) und mit Beispielen versehen sind die Kategorien folgendermaßen charakterisiert $(\mathrm{W}=$ werden-Passiv, $\mathrm{S}=$ sein-Passiv, Nnom = Subjektsnominativ, Ngen = Objektsgenitiv, Ndat = Objektsdativ, Npräp = Präpositionalkasus als Satzglied, pN = Präpositionalphrase mit dem besonderen Merkmal "Subjektfähigkeit", wobei N für Nomen, Nominalgruppe oder Pronomen steht):

I 1: $\mathrm{N}_{\text {nom }} \mathrm{V}_{\text {pass }} \mathrm{pN}$

W: Er wird von mir geschlagen.

S: Der Brief ist von mir geschrieben.

I 2: $\mathrm{N}_{\text {nom }} \mathrm{V}_{\text {pass }}$

W: Er wird geschlagen.

S: Der Brief ist geschrieben.

II 1: $\mathrm{N}_{\text {gen/dat/präp }} \mathrm{V}_{\text {pass }} \mathrm{pN}$

W: Der Toten wird von uns gedacht.

W: Dir wird von ihm geholfen.

W: Für Arbeit wird von mir gesorgt.

S: $\quad$ Für Arbeit ist von mir gesorgt.

II 2: $\mathrm{N}_{\text {gen/dat/präp }} \mathrm{V}_{\text {pass }}$

W: Der Toten wird gedacht. 
W: Dir wird geholfen.

W: Für Arbeit wird gesorgt.

S: Für Arbeit ist gesorgt.

III 1: $\mathrm{pN} \mathrm{V}_{\text {pass }}$

W: Von ihm wird getanzt.

III 2: $\mathrm{V}_{\text {pass }}$

W: Es wird getanzt.

Dabei fällt auf, dass Brinker kein sein-Passiv für Kategorie III ansetzt, also das unpersönliche Passiv intransitiver Verben ohne Objekt bzw. absolut gebrauchter transitiver Verben, da es in seinem Korpus offensichtlich nicht vorkommt. Im Chat-Korpus wird das einzige unpersönliche sein-Passiv aber gerade von einem solchen absolut gebrauchten transitiven Verb gebildet (s. Abschnitt 5). Für meine Untersuchung wird deshalb keine Kategorie von vornherein ausgeschlossen.

\section{Das persönliche Passiv}

Nachfolgend werden die Zahlen hinsichtlich des persönlichen bzw. subjekthaltigen Passivs nach werden- und sein-Passiv und zusätzlich jeweils nach \pm Agenshinzufügung verglichen. Einige Beispiele aus dem Chat-Korpus in der Notation von Brinker 1971 (s.o.):

I 1 W: Es reicht, jeden Tag von irgendwelchen Idioten angemacht zu werden, die sich beschweren, dass irgendwas nicht geht.

I 1 S: Dieser channel ist nur von einer sehr kleinen Münsteraner Truppe besetzt.

I 2 W: Ein channel wird kreiert, indem man ihn betritt.

I 2 S: $60 \%$ aller Lebensmittel im Supermarkt sind genetisch verändert.

Die Zahlen für das persönliche Passiv bei Schoenthal 1976 ergeben sich durch Abzug des unpersönlichen Passivs (a.a.O. 182). Da Schoenthal hinsichtlich der Agenshinzufügung (a.a.O. 183, 188) keine Angaben macht, inwieweit das unpersönliche Passiv daran beteiligt ist, wird davon ausgegangen, dass alle Fälle von Agenshinzufügung beim persönlichen Passiv auftreten. Da dies aber nicht sicher ist, werden die Angaben in Klammern gesetzt. Auf jeden Fall wären die Abweichungen sehr gering. 


\begin{tabular}{|c|c|c|l|}
\hline $\begin{array}{l}\text { persönliches werden- } \\
\text { Passiv }\end{array}$ & $\begin{array}{l}\text { Brinker (1971: } \\
\text { 39f.) }\end{array}$ & $\begin{array}{l}\text { Schoenthal (1976: 173, } \\
183)\end{array}$ & Chat \\
\hline Gesamt relativ & $\mathbf{6 4 \%}$ & $\mathbf{6 4 \%}$ & $\mathbf{6 2 \%}$ \\
\hline ohne Agens relativ & $\mathbf{8 6 \%}$ & $\mathbf{( 8 3 \% )}$ & $\mathbf{8 4 \%}$ \\
\hline mit Agens relativ & $14 \%$ & $(17 \%)$ & $16 \%$ \\
\hline
\end{tabular}

\begin{tabular}{|c|c|c|c|}
\hline persönliches sein-Passiv & $\begin{array}{c}\text { Brinker } \\
(1971: 82 \mathrm{f} .)\end{array}$ & $\begin{array}{c}\text { Schoenthal } \\
(1976: 173,188)\end{array}$ & Chat \\
\hline Gesamt relativ & $\mathbf{3 6 \%}$ & $\mathbf{3 6 \%}$ & $\mathbf{3 8 \%}$ \\
\hline ohne Agens relativ & $\mathbf{8 7 \%}$ & $\mathbf{( 9 1 \% )}$ & $\mathbf{9 1 \%}$ \\
\hline mit Agens relativ & $13 \%$ & $(9 \%)$ & $9 \%$ \\
\hline
\end{tabular}

Es fällt auf, dass im Chat-Korpus das sein-Passiv etwas stärker, das werden-Passiv etwas geringer vertreten ist als bei Brinker oder Schoenthal. Dabei ist interessant, dass Schoenthal (1976: 217f.) gerade in Hinblick auf private Kommunikationssituationen ein Überwiegen von sein- gegenüber werden-Passiven feststellen konnte. Es ist nun möglich, dass die Befunde aus dem Chat-Korpus eine Tendenz in diese Richtung wiedergeben. Allerdings bleibt grundsätzlich ungeklärt, warum unter nicht-öffentlichen Kommunikationsbedingungen das sein- Passiv häufiger sein soll als das werden-Passiv.

Weiter fällt auf, dass die Werte für +Agenshinzufügung beim werden-Passiv für das ChatKorpus zwischen Brinker und Schoenthal liegen, beim sein-Passiv dagegen exakt mit Schoenthal übereinstimmen. Aufgrund der geringen Differenzen ist dem jedoch keine besondere Bedeutung zuzumessen.

$\mathrm{Zu}$ erwarten ist außerdem, dass das Passiv in Fach- und Wissenschaftssprachen aufgrund der geringeren Relevanz des Handlungsträgers überdurchschnittlich häufig vertreten ist (vgl. Pape-Müller 1980: 147). So finden sich bei Brinker 1971 im Texttyp "wissenschaftliche und populärwissenschaftliche Literatur" etwa zwei Drittel aller Passivformen (62.3\% der werdenund $71.7 \%$ der sein-Passive; a.a.O. 39f., 82f.), ähnlich verhält es sich mit dem "technischen" Chatroom "Linux.de", auf den mit ca. 150 persönlichen Passiven allein $42 \%$ entfallen.

Auch hinsichtlich der Präposition in der Agenshinzufügung sind keine großen Diskrepanzen zu verzeichnen. Beim werden-Passiv nimmt den größten Teil die Präposition von ein, wobei meine Ergebnisse näher bei Schoenthal (a.a.O. 183, 187) als bei Brinker (a.a.O. 48) liegen. (Da weder Brinker noch Schoenthal hinsichtlich der Agenshinzufügung zwischen persönlichem und unpersönlichem Passiv unterscheiden, ist das unpersönliche Passiv bei ihnen jeweils mit eingerechnet. Da es sich dabei jedoch um sehr wenige bis gar keine Fälle handelt, dürften die Zahlen nahezu identisch sein.) 


\begin{tabular}{|c|c|c|c|}
\hline $\begin{array}{c}\text { persönliches werden- } \\
\text { Passiv }\end{array}$ & Brinker 1971 & Schoenthal 1976 & Chat \\
\hline Gesamt mit Agens & 1430 & 127 & 35 \\
\hline von-Anteil relativ & $\mathbf{4 3 \%}$ & $\mathbf{5 5 . 9 \%}$ & $\mathbf{6 0 \%}$ \\
\hline
\end{tabular}

\section{Das unpersönliche Passiv}

Auch hinsichtlich des unpersönlichen bzw. subjektlosen Passivs werden die Zahlen nach werden- und sein-Passiv und zusätzlich jeweils nach \pm Agenshinzufügung verglichen. Einige Beispiele in der Notation von Brinker 1971 (s.o.):

I 1 W: Also, wie kann ich es am besten basteln, dass beim disconnect vom Provider automatisch neu angewählt wird?

I 1 S: Kein Beispiel im Korpus.

I 2 W: Es wurde eingekauft.

I 2 S: Moment ... installiert ist also schon?

Da Schoenthal (1976: 183, 188) hier ebenfalls keine Angaben macht, inwieweit das unpersönliche Passiv an der Agenshinzufügung beteiligt ist, wird davon ausgegangen, dass alle Fälle von Agenshinzufügung beim persönlichen Passiv auftreten. Da dies aber nicht sicher ist, werden die Angaben in Klammern gesetzt.

\begin{tabular}{|c|c|c|c|}
\hline $\begin{array}{c}\text { unpersönliches werden- } \\
\text { Passiv }\end{array}$ & Brinker (1971: 39f.) & $\begin{array}{c}\text { Schoenthal } \\
(1976: 182)\end{array}$ & Chat \\
\hline Gesamt relativ & $\mathbf{3 \%}$ & $\mathbf{4 \%}$ & $\mathbf{1 1 \%}$ \\
\hline ohne Agens relativ & $98 \%$ & $(100 \%)$ & $96 \%$ \\
\hline mit Agens relativ & $2 \%$ & $(0) \%$ & $4 \%$ \\
\hline
\end{tabular}

\begin{tabular}{|c|c|c|c|}
\hline $\begin{array}{c}\text { unpersönliches sein- } \\
\text { Passiv }\end{array}$ & Brinker (1971: 82f.) & $\begin{array}{c}\text { Schoenthal } \\
(1976: 182)\end{array}$ & Chat \\
\hline Gesamt relativ & $\mathbf{0 . 0 5 \%}$ & $\mathbf{1 \%}$ & $\mathbf{1 \%}$ \\
\hline ohne Agens relativ & $94 \%$ & $(100 \%)$ & $100 \%$ \\
\hline mit Agens relativ & $6 \%$ & $(0 \%)$ & $0 \%$ \\
\hline
\end{tabular}

Während das unpersönliche sein-Passiv in allen drei Korpora kaum belegt ist, ergibt sich hinsichtlich des unpersönlichen werden-Passivs eine deutliche Diskrepanz für Chat mit $11 \%$ vs. 3 bzw. $4 \%$ bei Brinker und Schoenthal. Als Begründung lässt sich anführen, dass sich das unpersönliche Passiv durch eine besonders starke Situationsbindung auszeichnet (Weinrich 2003: 177), eine Eigenschaft, die mit dem Merkmal "situations- und handlungs[ge]bunden" korreliert, das konzeptionelle Mündlichkeit in Hinblick auf die Kommunikationssituation auszeichnet (in Anlehnung an Koch/Oesterreicher 1994: 588). Eine solche Situations- und Handlungsgebundenheit kann im Chat zumindest virtuell gegeben sein. Dem entspricht auch, 
dass bei Schoenthal 1976 der Texttypus Dialog (z.B. Diskussion), der dem Chat konzeptionell am nächsten kommt, mit immerhin 68\% am unpersönlichen Passiv beteiligt ist (a.a.O. 182).

Als nächstes soll untersucht werden, welche Unterkategorien des unpersönlichen Passivs vertreten sind. Vergleiche sind hier nur mit Brinker 1971 möglich, da Schoenthal 1976 keine weiteren Angaben dazu macht.

In Übereinstimmung mit Brinker 1971 wird ein Typus W/S II, d.h. ein werden- oder seinPassiv von Intransitiva mit Genitiv-, Dativ- oder Präpositionalobjekt, sowie ein Typus W/S III angesetzt. Für Typus III, der bei Brinker nicht weiter besprochen wird, ergeben sich noch weitere Differenzierungen in 1. reine Intransitiva ohne Objekt (z.B. es wird gelacht), 2. transitive Verben in absoluter Verwendung (d.h. mit nicht-ausgedrücktem Akkusativobjekt, z.B. es wird geküsst), und 3. intransitive Verben in absoluter Verwendung (d.h. mit nichtausgedrücktem Dativ-, Genitiv- oder Präpositionalobjekt, z.B. es wird geholfen).

In der Gruppe W/S II, also Intransitiva mit Objekt, finden sich fünf Beispiele, alles werdenPassive und alle ohne Agens (also ausschließlich W II 2 in Brinker-Notation). Dabei handelt es sich um drei Verben mit Präpositionalobjekt (spielen mit, hoffen auf, üben an) und um zwei Verben mit Dativobjekt. (kündigen, helfen).

Kommt drauf an, womit gespielt wird.

Also wird auf Kulanz der Beamten gehofft?

An dir wurde schon geübt, wie ich sehe.

Dir wurde gekündigt???

Bei konkreteren Fragen wird dir dann gerne geholfen.

Für das unpersönliche werden-Passiv von Intransitiva mit Objekt ergibt sich damit ein Anteil von nur 19\% im Chat-Korpus vs. 69\% bei Brinker 1971 (a.a.O. 39f.). Dementsprechend machen unpersönliche Konstruktionen ohne Objekt bei Brinker 1971 31\%,ํㅣ im Chat-Korpus dagegen $81 \%$ aus. Die auffällige Diskrepanz zwischen objekthaltigem und objektlosem unpersönlichen Passiv kommt durch die bereits weiter oben konstatierte starke Situationsbindung zustande, die v.a. für die objektlose Konstruktion gilt (Weinrich 2003: 177).

Bei einer weiteren Differenzierung des objektlosen unpersönlichen Passivs zeigt sich außerdem, dass die Transitiva in absoluter Verwendung mit 57\% den größten Anteil ausmachen.

\footnotetext{
${ }^{1}$ Dies bestätigt sich an einer Aufstellung der Passivformen in "Unkenrufe" von Günter Grass, die mir Sari Valkama-Ikonen von der Universität Jyväskylä freundlicherweise zur Verfügung gestellt hat. Dort sind es 38\% objektlose versus $62 \%$ objekthaltige unpersönliche Passive, wobei in letzterer Kategorie fast ausschließlich Präpositionalobjekte vorkommen.
} 


\begin{tabular}{|l|c|c|c|c|}
\hline unpersönliches & mit & \multicolumn{2}{|c|}{ ohne Objekt oder Objekt unausgedrückt } \\
\cline { 3 - 5 } werden-Passiv & Objekt & $\begin{array}{c}\text { 1. reine } \\
\text { Intransitiva }\end{array}$ & $\begin{array}{c}\text { 2. Transitiva in } \\
\text { absoluter } \\
\text { Verwendung }\end{array}$ & $\begin{array}{c}\text { 3. Intransitiva in } \\
\text { absoluter } \\
\text { Verwendung }\end{array}$ \\
\hline Gesamt relativ & $\mathbf{1 9 \%}$ & $29 \%$ & $57 \%$ & $14 \%$ \\
\cline { 3 - 5 } & & \multicolumn{3}{|c}{} \\
\hline
\end{tabular}

\section{Reine Intransitiva ohne Objekt}

Und wenn das Paket richtig schwer ist, wird gar nicht erst in den 4ten Stock gelaufen.

Es wird [...], weggeguggt, [...].

Schaut mal rum, ob irgendwo gequized wird.

Da wird gequizzed?

Da wird geballert und geballert.

Besonders, da einigermaßen schön weit gefasst werden kann.

\section{Transitiva in absoluter Verwendung (Auswahl)}

Die Küche ist sauber, da wird nie wieder drin gekocht.

Heute muss ich mir mal nichts bestellen ... es wurde eingekauft.

Klar wird da gespielt.

Wie kann ich es am besten basteln, dass beim disconnect vom Provider automatisch neu angewählt wird?

Hier wird nicht geknufft.

In Deliland wird geraucht??

Und genau dafür wird gebastelt.

\section{Intransitiva in absoluter Verwendung}

Tja, pass man einmal nich auf, [da] wird hinter dem Rücken ohne Ende geredet. (reden über) Es wird vorbeigefahren, [...], [...]. (vorbeifahren an)

Eingebrochen wurde auch noch nicht. (einbrechen in)

Was den Vergleich unpersönliches sein-Passiv versus werden-Passiv angeht, so ist ersteres extrem selten. Im Chat-Korpus kommt nur ein Beispiel aus dem Bereich S III 2 vor, genauer: ein transitives Verb im absoluten Gebrauch ohne Agens. Dies entspricht $1 \%$ aller seinPassive.

Moment ... installiert ist also schon?

Bei Schoenthal (1976: 182; keine Angaben zur Agenshinzufügung) sind es 3 Beispiele, das entspricht ebenfalls 1\%. Brinker (1971: 82f.) zählt immerhin 17 Fälle, allerdings nur im Bereich II (Intransitiva mit Objekt), das sind $0.5 \%$ der sein-Passive (davon 1 Fall mit Agens). Wie bereits in Abschnitt 3 erwähnt, setzt Brinker (1971: 71) keine Kategorie S III an, d.h. ein unpersönliches sein-Passiv von Verben ohne Objekt existiert in seinem Korpus nicht. Umgekehrt kommt im Chat-Korpus kein Passiv vom Typ S II vor, d.h. ein unpersönliches Passiv von Intransitiva mit Objekt. Aufgrund der geringen Vorkommen an sein-Passiven wird hier nicht weiter auf diesen Konstruktionstyp eingegangen. 
Andere unpersönliche Passive kommen im Chat-Korpus nicht vor. Theoretisch möglich wären noch Reflexivpassive, die v.a. der Umgangs- und damit konzeptionell mündlicher Sprache zugewiesen werden (etwa Duden 1998: 180). Da sie aber typischerweise "energische Aufforderungen" ausdrücken (z.B. Jetzt wird sich gewaschen!, a.a.O.), ist ihr Anwendungsbereich extrem eingegrenzt.

Ähnliches gilt für Passive mit Numerus-Inkongruenz, die z.B. auftritt, wenn das finite Verb im Singular, das Subjekt im Plural steht, z.B. Es wurde Karten gespielt. Im Falle einer solchen Inkongruenz wird von manchen Autoren (z.B. Abraham 1986 und Walther 1990) sogar davon ausgegangen, dass hier kein Nominativ und damit ein Subjekt, sondern ein Akkusativ und damit ein Objekt vorliegt. Walther (1990: 93) nimmt außerdem an, dass solche Konstruktionen in der Umgangssprache durchaus akzeptabel seien. Sie nennt u. a. folgende (allerdings nicht belegte) Beispiele.

Lieder gesungen wird (von Kindern) gern.

Hier wird keine Faxen gemacht!

Im Chat-Korpus lassen sich derartige Vorkommen nicht finden.

Ein Satz aus dem Korpus wirft allerdings die Frage auf, ob es nicht doch Passive mit beibehaltenem Akkusativobjekt gibt. Es handelt sich um eine Konstruktionen, in der einem maskulinen Substantiv der indefinite Artikel nen vorausgeht:

Und wenn das Paket zu schwer ist, wird nen Zettel an die Tür geklebt, dass man das Paket abholen kann.

Da nen sprechsprachlich i. Allg. als verkürzte Form des Akkusativs einen interpretiert werden kann (Ich hab nen Stift.) würde der Satz in seiner "Vollform" so lauten:

Und wenn das Paket zu schwer ist, wird einen Zettel an die Tür geklebt, dass man das Paket abholen kann.

Allerdings tritt dieselbe Form nen auch mit maskulinen Substantiven im Nominativ bzw. in einem Fall mit einem neutralen Substantiv im Akkusativ auf, wobei grundsätzlich in allen Beispielen ein (und nicht einen) ersetzt wird.

Ich seh aus wie nen Waldschrat.

Bissu nen der oder ne sie?

Wäre das nicht sowieso nen Horror, wenn zwei Bottiche reden würden?

Ich denk mal, dass das, trotzdem dass du keine Lust hast, nen ganz netter Abend wird.

War recht interessant, und ist nen Grund, dich auf ignore zu setzen.

Will jemand nen Glas Ökosoja?

Meine Vermutung ist deshalb, dass die umgangssprachliche Variante $n$, die ein sowohl im Nominativ als auch im Akkusativ ersetzen kann (Ich hab $n$ Auto, Das is $n$ Auto), von manchen Sprechern als zu kurz empfunden und deshalb zu nen "aufgefüllt" wird. Dadurch fällt sie automatisch mit der verkürzten Form des Akkusativs nen (= einen) zusammen. Burri (in diesem Band) spricht hier auch von einer erweiterten Kurzform. Ein Vorkommen des 
indefiniten Artikels nen im Passiv ist damit aber kein Hinweis auf einen Akkusativ und so auch nicht auf ein unpersönliches Passiv mit beibehaltenem Akkusativobjekt.

\section{Exkurs zum "Platzhalter"-es}

Gerade im Zusammenhang mit dem unpersönlichen Passiv wird häufiger das "Platzhalter"-es als typisch genannt, d.h. ein es, das keine Satzgliedfunktion hat, sondern im Aussagesatz bei Fehlen eines anderen Satzgliedes die erste Stelle vor dem finiten Verb einnimmt und bei Permutation wegfällt (vgl. Duden 1998: 175f.).

Es wird ihm geholfen.

Ihm wird geholfen.

Hat der entsprechende Satz ein Subjekt, so befindet sich dieses in rhematischer Position, ist typischerweise indefinit und kongruiert mit dem finiten Verb.

Es werden Lieder gesungen.

Heute werden Lieder gesungen.

Schon Schoenthal (1976: 120, Fußnote 213) kritisiert, dass man in der Literatur zum Passiv (z.B. Helbig 1968: 131) den Eindruck bekomme, dass dieses es speziell ein Merkmal des unpersönlichen Passivs sei. Sie nimmt dagegen an (a.a.O. 120), dass es "offensichtlich in jedem Satz die erste Position einnehmen kann". Ihre drei nachfolgenden Beispiele sind dann zwar subjekthaltig, aber immer noch Passive. Auch Brinker (1971: 36) spricht davon, dass ein solches es besonders beim subjektlosen Passiv auftrete, obwohl auch er Beispiele für das persönliche Passiv mit dem "Platzhalter"-es nennt (a.a.O.). Eine Auszählung erfolgt allerdings weder bei Brinker noch bei Schoenthal.

Im Chat-Korpus finden sich 19 Vorkommen eines solchen "Platzhalter"-es (dabei kommen in einem Satz drei unpersönliche Passive in asyndetischer Reihung vor, die als drei Vorkommen gezählt werden.). Es zeigt sich allerdings, dass den größten Anteil gerade nicht Passiv-, sondern Aktivsätze auf sich vereinen. Sie sind mit $68 \%$ beteiligt, während das unpersönliche Passiv 21\% und das persönliche Passiv 11\% ausmachen. Damit ist eindeutig gezeigt, dass das "Platzhalter"-es primär Aktivsätze und nicht Passivsätze auszeichnet, wobei es auch dort nicht ausschließlich im unpersönlichen Passiv angesiedelt ist.

\begin{tabular}{|c|c|c|c|}
\hline "Platzhalter"-es & Aktiv & $\begin{array}{c}\text { Unpersönliches } \\
\text { Passiv }\end{array}$ & Persönliches Passiv \\
\hline Gesamt relativ & $\mathbf{7 0 \%}$ & $\mathbf{2 1 \%}$ & $\mathbf{1 1 \%}$ \\
\hline
\end{tabular}

Aktiv (Auswahl)

Es sind unendliche, nichtperiodische Zahlen dabei.

Es fühlen sich sofort die richtigen angesprochen.

Es geschehen noch Zeichen und Wunder.

Es passen ja nicht unendlich viele Punkte aufs Papier.

Es röhren hier 2 Computer, 1 Laserdrucker und dann noch der Switch.

Ja schrei mal schön, es wird dir keiner helfen. 


\section{Unpersönliches Passiv}

Heute muss ich mir mal nichts bestellen ... es wurde eingekauft.

Es wird vorbeigefahren, weggeguggt, ignoriert.

\section{Persönliches Passiv}

Es sind ja auch alle IRC-Operatoren, die von der Server-Konsole ircen wollen, gebant.

Es wird ein device erstellt, auf das man dann den pppd setzt, fertig.

Dass es sich dabei nicht um ein spezifisches Merkmal konzeptionell mündlicher Texte handelt, wird durch eine exemplarische Untersuchung an einem literarischen Text, nämlich "Unkenrufe" von Günter Grass, bestätigt. Hier kommt das "Platzhalter"-es zu 75\% im Aktiv, zu 25\% im persönlichen Passiv vor, das unpersönliche Passiv ist überhaupt nicht beteiligt.

\section{Passivvarianten}

Als Passivvarianten können solche Konstruktionen bezeichnet werden, die rein äußerlich nicht dem "kanonischen" Passiv mit werden/sein + Partizip Perfekt entsprechen, aber gewisse Ähnlichkeiten mit diesem aufweisen. Im Folgenden wird innerhalb der Passivvarianten zwischen dem Dativpassiv und den Passivperiphrasen unterschieden.

Das Dativpassiv (z.B. Eroms 1978: 359) wird auch als Adressaten-Passiv (z.B. Schlobinski 1992: 173), Rezipienten-Passiv (z.B. Eisenberg 2001: 132; Reis 1985) oder bekommen-Passiv (z.B. Leirbukt 1997; Zifonun et al. 1997: 1824) bezeichnet. Es wird mit bekommen, erhalten oder kriegen + Partizip Perfekt gebildet: Wir bekommen das Buch geschenkt. Das Partizip kann dabei nur mit Verben gebildet werden, die im Aktiv einen Dativ der Person und einen Akkusativ der Sache bei sich haben, wobei der Dativ zum Subjekt des Passivsatzes wird und der Akkusativ beibehalten wird. Der Status des Dativ-Passivs ist teilweise immer noch umstritten, was auch daran liegt, dass es stark umgangssprachlichen Charakter hat und außerdem weitgehend auf den Süden des deutschen Sprachgebiets beschränkt zu sein scheint (Pape-Müller 1980: 37, auch Fußnote 26).

Aufgrund dieses umgangssprachlichen Status des Dativpassivs wäre zu erwarten, dass es in konzeptionell mündlicher Sprache und damit auch im Chat häufiger auftritt. Dies bestätigt sich dahingehend, dass sowohl das Schoenthal- als auch das Chat-Korpus einen höheren Anteil zeigen als das Brinker-Korpus, wobei das Dativpassiv im Chat-Korpus sogar noch häufiger vorkommt als bei Schoenthal. Eine Beschränkung auf den Süden Deutschlands ist aber gerade nicht (mehr?) festzustellen, da das Dativpassiv zu fast $70 \%$ in den norddeutschen City Channels "Berlin", "Hamburg" und "Münster" vorkommt.

\begin{tabular}{|c|c|c|c|}
\hline Dativpassiv & $\begin{array}{c}\text { Brinker } \\
(1971: 119)\end{array}$ & $\begin{array}{c}\text { Schoenthal } \\
(1976: 214)\end{array}$ & Chat \\
\hline $\begin{array}{c}\text { Verhältnis des Dativpassivs } \\
\text { zum Gesamtpassiv }\end{array}$ & $\mathbf{0 . 3 \%}$ & $\mathbf{1 \%}$ & $\mathbf{3 . 4 \%}$ \\
\hline
\end{tabular}


Hinsichtlich der drei Hilfsverben bekommen, kriegen und erhalten stellt Eroms (1978: 359368) fest, dass kriegen das ältere sei (so auch die ältesten Beispiele aus dem 16./17. Jahrhundert, die mit kriegen gebildet sind), während bekommen und erhalten erst im 19. Jahrhundert dazukommen (a.a.O. 366). Etwa im 18. Jahrhundert tritt kriegen als Vollverb aber in der Schriftsprache gegenüber bekommen und erhalten zurück, während es im Mündlichen erhalten bleibt (a.a.O. 363). Dies wirkt sich auch auf das Dativpassiv aus, wo die Variante mit kriegen inzwischen als die umgangssprachliche und niederere empfunden und in öffentlichen Kommunikationssituationen vor allem von bekommen abgelöst wird (a.a.O. 360). Schlobinski (1992: 178) verzeichnet dementsprechend für sein Korpus des Gegenwartsdeutschen ein Übergewicht der kriegen-Variante in der Sprechsprache bzw. der bekommen-Variante in der Schriftsprache.

\begin{tabular}{|c|c|c|}
\hline & Sprechsprache & Schriftsprache \\
\hline kriegen & $\mathbf{9 8 . 7 \%}$ & $8.6 \%$ \\
\hline bekommen & $11.3 \%$ & $\mathbf{7 7 . 3 \%}$ \\
\hline
\end{tabular}

Diesem Befund widersprechen allerdings die Daten aus dem Chat- sowie dem SchoenthalKorpus, da die bekommen-Variante hier überwiegt.

\begin{tabular}{|c|c|c|c|}
\hline Dativpassiv & $\begin{array}{c}\text { Schoenthal } \\
(1976: 214)\end{array}$ & Chat & Schlobinski (1992: 179) \\
\hline bekommen & $\mathbf{8 9 \%}$ & $\mathbf{6 9 \%}$ & $12 \%$ \\
\hline kriegen & $11 \%$ & $31 \%$ & $\mathbf{8 8 \%}$ \\
\hline
\end{tabular}

Eine Erklärung für dieses Phänomen steht noch aus. Immerhin gilt bekommen aber als stilistisch neutral (z.B. Hentschel/Weydt 1995: 172) so dass zumindest nicht ausgeschlossen ist, dass es auch in konzeptionell mündlicher Sprache gehäuft auftritt. Das bestätigt auch Leirbukt (1997: 111): "Es kann ferner festgestellt werden, daß bekommen entsprechend seinem stilistisch neutralen Charakter (auch) zur Wiedergabe gesprochener Sprache recht häufig benutzt wird."

Nachfolgend sind alle Beispiele aus dem Korpus verzeichnet (wobei allein schenken drei Mal vorkommt).

\section{kriegen}

Ich dachte, das kriegt man mit windows geliefert.

Du kriegst beides in einem, den t-online account und das Teil, zugeschickt.

Der kriegt $n$ frisches Bier ins Hemd gegossen.

Du kriegst also unter deiner kompletten Info angezeigt, wer hier männlich und wer weiblich ist?

\section{bekommen}

Ich hab heute 3.50 DM ausgezahlt bekommen.

Und es gibt keine schärferen Kontrollen, was die Viecher alles gespritzt bekommen. 
Hab grad kürzlich von el cheffe $n$ kleinen Terminplaner zu Weihnachten geschenkt bekommen.

Hab neulich ein Buch geschenkt bekommen: "[...]".

Nope, geschenkt bekommen.

Jetzt habe ich auf meine Frage hin eine Software auf den Tisch geknallt bekommen.

Weil ich heut Abend irgendwie ein Dauerkick von Peer verpasst bekomme.

Hab von Delia sogar schon mal ne Zigarette angeboten bekommen.

Hast du eigentlich deine Netzwerkkarte gratis geschickt bekommen?

Obwohl nicht zu den Passivvarianten gehörig, soll abschließend noch eine formal ähnliche Konstruktion (bekommen + Objekt + Partizip Perfekt) aus dem Chat-Korpus erwähnt werden, die Hentschel/Weydt (1995: 171) "modales Aktiv" nennen. In diesem Fall hat das Subjekt agentische Semantik und die ganze Konstruktion eine modale Komponente im Aktiv: Ich bekomme die Nüsse nicht geknackt $\approx$ Ich kann die Nüsse nicht knacken. M.E. handelt es sich um eine Konstruktion, die weitgehend der konzeptionell mündlichen oder Umgangssprache angehört, was sich auch daran zeigt, dass das modale Aktiv im Chat-Korpus insgesamt acht Mal vertreten ist. Interessanterweise überwiegt hier kriegen mit sechs Belegen (entspricht 75\%) über bekommen. Hentschel/Weydt (1995: 172) halten es für möglich, dass dies damit zusammenhängt, dass kriegen als Vollverb die Tendenz hat, sein Subjekt zu agentivieren, was für bekommen und erhalten nicht zu gelten scheint, z.B.: Ich werd dich schon noch kriegen! (*Ich werd dich schon noch bekommen!, *Ich werd dich schon noch erhalten!). Man vergleiche dazu auch Abraham (1985: 149), der hinsichtlich verwandter Konstruktionen mit Adjektiv statt mit Partizip Perfekt (Ich kriege die Mäuler satt) feststellt, dass das Subjekt zu kriegen hier ein Agens und kein Rezipient ist, und der Periphrase "es schaffen, es so weit kriegen" entspricht. Damit ließe sich das Überwiegen von kriegen beim modalen Aktiv erklären.

Nachfolgend einige Beispiele aus dem Chat-Korpus.

\section{bekommen}

Wie bekomm ich mein cdrom gemounted?

Ich bekomme das ns558 nicht geladen.

\section{kriegen}

Wir kriegen das schon erklärt.

Wieso krieg ich mich nicht wieder zurückbenannt?

Dummerweise kriege ich den nicht mal auf x86 compiled.

$\mathrm{Zu}$ den Passivvarianten können neben dem Dativpassiv auch noch die Passivperiphrasen gezählt werden. Sie zeichnen sich durch Akkusativkonversion aus (im Gegensatz zur Dativkonversion beim Dativpassiv) und haben aktivische Form, aber passivische Bedeutung mit meist zusätzlicher modaler Komponente, weshalb sie in ein werden-Passiv mit dem Hilfsverb können, müssen oder sollen überführt werden können. Die wichtigsten Passivperiphrasen im Deutschen sind die folgenden (vgl. Hentschel/Weydt 1994: 126; zu einer detaillierteren Übersicht aller Passivperiphrasen im Gegenwartsdeutschen vgl. auch Askedal 1987: 22-32): 
a) sein $+z u+$ Infinitiv mit können- oder müssen/sollen-Modalität: Diese Aufgabe ist zu lösen $\approx$ Diese Aufgabe kann bzw. muss/soll gelöst werden. sein $+z u+$ Infinitiv wird auch als modaler Infinitiv (z.B. Gelhaus 1977) bezeichnet.

b) lassen + sich + Infinitiv mit können-Modalität: Das Problem lässt sich auf verschiedene Weisen lösen $\approx$ Das Problem kann auf verschiedene Weisen gelöst werden.

c) Reflexivkonstruktionen mit können-Modalität: Das Buch liest sich leicht $\approx$ Das Buch kann leicht gelesen werden.

a) Die Passivperiphrase sein $+z u+$ Infinitiv wird auch bei Brinker (1971: 121-123) und Schoenthal (1976: 213) zahlenmäßig untersucht. Dabei fällt auf, dass die Konstruktion dort sehr viel häufiger ist als im Chat-Korpus. Es ist möglich, dass es sich dabei um eine Form handelt, die eher in öffentlichen Kommunikationssituationen vorkommt. Dies scheint zumindest für die müssen/sollen-Variante zu gelten, die typisch für Texte mit Vorschriftscharakter (z.B. juristische Texte) und für wissenschaftliche Literatur ist (PapeMüller 1980: 182).

Nachfolgend die Ergebnisse aus den drei Korpora (wobei Brinker nur einen Teil des Materials mit ca. 100.000 Finita auswertet (a.a.O. 123; 147, Fußnote 229)).

\begin{tabular}{|c|c|c|c|}
\hline sein $+z u+$ Infinitiv & Brinker 1971 & $\begin{array}{c}\text { Schoenthal } \\
1976\end{array}$ & Chat \\
\hline Anteil an den Gesamtfinita & $\mathbf{0 . 4 \%}$ & $\mathbf{0 . 7 \%}$ & $\mathbf{0 . 1 \%}$ \\
\hline
\end{tabular}

Da die Konstruktion sein $+z u+$ Infinitiv sowohl eine können- als auch eine müssen/sollenModalität haben kann (s.o.), stellt sich die Frage, in welchem zahlenmäßigen Verhältnis diese zueinander stehen. In Brinkers (eingeschränktem) Korpus ist die können-Modalität zu 71\%, die müssen/sollen-Modalität zu 21\% vertreten (a.a.O. 122f.; keine Angabe zu den restlichen 8\%). Mit $83 \%$ können- und 17\% müssen/sollen- Modalität im Chat-Korpus ergeben sich in der Hinsicht kaum Unterschiede.

In diesem Zusammenhang ist außerdem interessant, dass eine Konstruktion im Korpus auftaucht, die von vielen Autoren als höchst unwahrscheinlich oder sogar als unmöglich ausgeschlossen wird (z.B. Askedal 1987: 23f.; Brinker 1971: 121; Gelhaus 1977: 172, 176 nach Pape-Müller 1980: 176). Es handelt sich dabei um die Kombination von sein $+z u+$ Infinitiv mit können-Modalität und einer Agenshinzufügung mit der Präposition von, die sonst nur mit der müssen-Modalität möglich scheint (vgl. z.B. Pape-Müller 1980: 178):

Naja, vielleicht haben die auch so ein verrücktes script wie bei uns, mail-weiterleiten heißt das, [das] ist sogar von nem Geisteswissenschaftler zu bedienen und macht nix als ne forward zu editieren.

Nachfolgend einige modale Infinitive aus dem Chat-Korpus. 


\section{können-Modalität}

[Das] Ist nicht zu erwarten.

[Das] ist sogar von nem Geisteswissenschaftler zu bedienen .

[Das] ist ja nicht auszuhalten

\section{müssen/sollen-Modalität}

Ist da viel einzustellen?

Realitätsverlust bei Internet-Backups zu befürchten!

Alle wissen, was zu tun ist.

b) Die Fügung lassen + sich + Infinitiv ist dann als Passivperiphrase mit können-Modalität zu interpretieren, wenn ein unbelebtes Subjekt auftritt: Das Problem lässt sich auf verschiedene Weisen lösen $\approx$ Das Problem kann auf verschiedene Weisen gelöst werden. Ist das Subjekt belebt, bekommt lassen die Interpretation von veranlassen oder zulassen und ist keine Passivperiphrase (Die Frau lässt sich tragen $\approx$ *Die Frau kann getragen werden; vgl. Brinker 1971: 119).

Brinker (1971: 121) untersucht auch hier wieder nur ein eingeschränktes Korpus, wobei der Anteil der Fügung an den Gesamtfinita eindeutig höher ist als im Chat-Korpus. Es ist möglich, dass auch für diese Passivperiphrase gilt, dass sie in öffentlicher Kommunikation häufiger ist.

\begin{tabular}{|c|c|c|}
\hline lassen + sich + Infinitiv & Brinker 1971 & Chat \\
\hline Anteil an den Gesamtfinita & $\mathbf{0 . 2 \%}$ & $\mathbf{0 . 0 3 \%}$ \\
\hline
\end{tabular}

Aber das, was ich mache, lässt sich halt nicht [mit] einem Wort oder gar einer V-Kartenzeile beschreiben.

Natürlich lässt sich das prima automatisieren.

Für eine feste Strecke lässt sich so etwas iirc [= sicher?] auch mit Lasern machen.

Lässt sich das nicht mit gated oder routed realisieren?

Es [= das Email-Programm] lässt sich nicht nachinstallieren.

Dabei ist sogar eines der sechs Beispiele unpersönlich, d.h. subjektlos, mit Präpositionalobjekt konstruiert:

OK, über Geschmack lässt sich nicht streiten.

c) An Reflexivkonstruktionen mit modaler können-Komponente als Passivperiphrase (Das Buch liest sich leicht $\approx$ Das Buch kann leicht gelesen werden) weist das Chat-Korpus nur drei Beispiele auf. Ein Vergleich ist weder mit Brinker noch mit Schoenthal möglich, da diese Konstruktionen bei ihnen nicht untersucht werden.

Aber es [= das Lied] singt sich super.

Keine Ahnung ob das Ding gut ist. [Es] liest sich aber gut.

Warum finden sich die dummen PCs nimmer seit ich das Kabel in nen Kabelkanal gelegt $h a b$ ? 
An sonstigen Passivperiphrasen tauchen im Chat-Korpus noch zwei auf, die hier nur der Vollständigkeit halber genannt seien.

- gehören + Partizip Perfekt, das müssen/sollen-Modalität hat und stark umgangssprachlich ist (vgl. Askedal 1987: 26):

Sorry, aber manchmal gehörst du aus dem Verkehr gezogen.

- bleiben + Partizip Perfekt, das dem sein-Passiv ähnelt, aber aufgrund von bleiben die kontinuative Aktionsart betont (vgl. Askedal 1987: 23):

Da bleibt keine Aorta geschlossen.

\section{Zusammenfassung}

Hinsichtlich der Zuordnung von Chattexten zu konzeptioneller Schriftlichkeit oder Mündlichkeit anhand des Phänomens Passiv ergibt sich als Ergebnis, dass der Chat tatsächlich eindeutig dem Pol "konzeptionelle Mündlichkeit" angehört. Die Daten aus dem Chat-Korpus liegen in allen Fällen näher bei den Werten aus dem Schoenthal-Korpus (das eine Zwischenstellung hinsichtlich konzeptioneller Schriftlichkeit/Mündlichkeit einnimmt) als bei denen aus dem Brinker-Korpus (das konzeptionelle Schriftlichkeit repräsentiert).

Als Merkmale konzeptioneller Mündlichkeit im Bereich von Passivkonstruktionen kristallisierte sich dabei zum einen die relative Häufigkeit von unpersönlichen Passiven heraus. Hier war allerdings auffällig, dass Bildungen von Intransitiva mit Objekt (z.B. mir wird geholfen) im Vergleich zu objektlosen Konstruktionen sehr selten sind. Dies wurde auf das Merkmal "kommunikativ bedingte Situationsgebundenheit" zurückgeführt, das sowohl ein Charakteristikum des objektlosen unpersönlichen Passivs als auch der Chat-Texte ist (hier zumindest im virtuellen Sinne). Außerdem ergab eine detaillierte Untersuchung, dass dabei transitive Verben in absoluter Verwendung eindeutig überwiegen, d.h. Konstruktionen mit nicht-ausgedrücktem Akkusativobjekt wie Ab und zu muss hier mal aufgeräumt werden.

Eine relativ häufigeres Auftreten im Vergleich zu konzeptionell weniger mündlichen Texten wurde auch im Bereich des Dativpassivs festgestellt, das ohnehin als stark umgangssprachlich gilt. Überraschend war allerdings der Befund, dass im Chat- wie im Schoenthal-Korpus die bekommen-Variante deutlich über die kriegen-Variante dominiert, obwohl einschlägige Untersuchungen genau umgekehrte Verhältnisse für konzeptionelle Mündlichkeit zeigen. Eine Erklärung dafür steht noch aus.

Darüberhinaus konnte der in der einschlägigen Literatur immer wieder suggerierte enge Zusammenhang zwischen dem unpersönlichen Passiv und dem „Platzhalter“-es widerlegt werden, da dieses sogar überwiegend bei Aktivsätzen auftritt. Exemplarische Auszählungen an einem literarischen Text und damit im Bereich konzeptionell schriftlicher Sprache bestätigten dieses Ergebnis. Es handelt sich also nicht um ein Merkmal konzeptionell mündlicher Sprache. 


\section{Literaturangaben}

Abraham, Werner (1985): "Grammatik von kriegen und bekommen". In: Redder, Angelika (ed.): Deutsche Grammatik II. Osnabrück: 142-165.

Abraham, Werner (1986): "Die Passivdiathese im Deutschen: Typen, Theorie". In: Burkhardt, Armin/Körner, Karl-Hermann (eds.): Pragmantax. Akten des 20. Linguistischen Kolloquiums, Braunschweig 1985. Tübingen: 15-24.

Askedal, John Ole (1987): "Syntaktische Symmetrie und Asymmetrie im Bereich der passivischen Fügungen des Deutschen". In: Das Passiv im Deutschen. Akten des Kolloquiums über das Passiv im Deutschen, Nizza 1986. Tübingen: 17-49.

Brinker, Klaus (1971): Das Passiv im heutigen Deutsch. Form und Funktion. München (= Heutiges Deutsch, Reihe I, 2).

Duden-Grammatik der deutschen Gegenwartssprache (1998). 6., neu bearbeitete Auflage. Mannheim etc. (= Der Duden 4).

Dürscheid, Christa (2002): Einführung in die Schriftlinguistik. Wiesbaden (= Studienbücher zur Linguistik 8).

Eisenberg, Peter (2001): Grundriß der deutschen Grammatik. Band 2: Der Satz. Unveränderte Neuauflage. Stuttgart.

Eroms, Hans-Werner (1978): "Zur Konversion der Dativphrasen". Sprachwissenschaft 3: 357405.

Gelhaus, Hermann (1977): Der modale Infinitiv. Tübingen. (= Forschungsberichte des Instituts für deutsche Sprache 35).

Grass, Günter (1992): Unkenrufe. Göttingen.

Helbig, Gerhard (1968): "Zum Problem der Genera des Verbs in der deutschen Gegenwartssprache". Deutsch als Fremdsprache 5: 129-148.

Hentschel, Elke/Weydt, Harald (1994): Handbuch der deutschen Grammatik. 2., durchgesehene Auflage. Berlin/New York.

Hentschel, Elke/Weydt, Harald (1995): "Das leidige bekommen-Passiv". In: Popp, Heidrun (ed.): Deutsch als Fremdsprache. An den Quellen eines Faches. München: 165-183.

Kilian, Jörg (2001): "T@stentöne. Geschriebene Umgangssprache in computervermittelter Kommunikation. Historisch-kritische Ergänzungen zu einem neuen Feld der linguistischen Forschung". In: Beißwenger, Michael (ed.): Chat-Kommunikation. Sprache, Interaktion, Sozialität \& Identität in synchroner computervermittelter Kommunikation. Perspektiven auf ein interdisziplinäres Forschungsfeld. Stuttgart: 55-78.

Koch, Peter/Oesterreicher, Wulf (1994): "Schriftlichkeit und Sprache (Writing and Language)". In: Günther, Hartmut/Ludwig, Otto (eds.): Schrift und Schriftlichkeit/Writing and its Use. Berlin/New York: 587-604.

Koch, Peter/Oesterreicher, Wulf (1985): "Sprache der Nähe - Sprache der Distanz. Mündlichkeit und Schriftlichkeit im Spannungsfeld von Sprachtheorie und Sprachgeschichte". Romanistisches Jahrbuch 36: 15-43.

Leirbukt, Oddleif (1997): Untersuchungen zum bekommen-Passiv im heutigen Deutsch. Tübingen (= Reihe Germanistische Linguistik 177). 
Ochs, Elinor (1979): "Planned and unplanned discourse". In: Givón, Talmy (ed.): Discourse and syntax. New York: 51-80.

Pape-Müller, Sabine (1980): Textfunktionen des Passivs. Tübingen (= Reihe Germanistische Linguistik 29).

Reis, Marga (1985): "Mona Lisa kriegt zu viel - Vom sogenannten ,Rezipientenpassiv“ im Deutschen". Linguistische Berichte 96: 140-155.

Schlobinski, Peter (1992): Funktionale Grammatik und Sprachbeschreibung. Eine Untersuchung zum gesprochenen Deutsch sowie zum Chinesischen. Opladen.

Schoenthal, Gisela (1976): Das Passiv in der deutschen Standardsprache. München (= Heutiges Deutsch, Reihe I, 7).

Schwitalla, Johannes (1997): Gesprochenes Deutsch. Eine Einführung. Berlin (= Grundlagen der Germanistik 33).

Storrer, Angelika (2001): "Getippte Gespräche oder dialogische Texte? Zur kommunikationstheoretischen Einordnung der Chat-Kommunikation". In: Lehr, Andrea et al. (eds.): Sprache im Alltag. Beiträge zu neuen Perspektiven in der Linguistik. Berlin/New York: 439-465.

Walther, Gisela (1990): "Syntax des unpersönlichen Passivs im Deutschen". In: Steube, Anita (ed.): Syntaktische Repräsentationen mit leeren Kategorien und Proformen und ihre semantischen Interpretationen. Berlin: 91-108.

Weinrich, Harald (2003): Textgrammatik der deutschen Sprache. 2. rev. Auflage. Mannheim usw.

Zifonun, Gisela/Hoffmann, Ludger/Strecker, Bruno (1997): Grammatik der deutschen Sprache. Band 3. Berlin/New York (= Schriften des Instituts für deutsche Sprache 7.3). 